\title{
Carbon Nanofibers-Poly-3-hydroxyalkanoates Nanocomposite: Ultrasound-Assisted Dispersion and Thermostructural Properties
}

\author{
A. M. Gumel, ${ }^{1}$ M. S. M. Annuar, ${ }^{1}$ K. A. Ishak, ${ }^{1}$ and N. Ahmad ${ }^{2}$ \\ ${ }^{1}$ Institute of Biological Sciences, Faculty of Science, University of Malaya, 50603 Kuala Lumpur, Malaysia \\ ${ }^{2}$ Department of Chemistry, Faculty of Science, University of Malaya, 50603 Kuala Lumpur, Malaysia \\ Correspondence should be addressed to A. M. Gumel; gumel@um.edu.my
}

Received 16 April 2014; Revised 14 July 2014; Accepted 16 July 2014; Published 3 August 2014

Academic Editor: John Z. Guo

Copyright (c) 2014 A. M. Gumel et al. This is an open access article distributed under the Creative Commons Attribution License, which permits unrestricted use, distribution, and reproduction in any medium, provided the original work is properly cited.

\begin{abstract}
The conductivity and high surface-to-volume ratio of carbon nanofibers (CNFs) composited with the medium-chain-length poly3-hydroxyalkanoate (mcl-PHA) have attracted much attention as smart biomaterial. However, poor CNF dispersion leads to tactoid agglomerated composite with poor crystallite morphology resulting in inferior thermomechanical properties. We employed acoustic sonication to enhance the construction of exfoliated PHA/CNFs nanocomposites. The effects of CNF loading and the insonation variables (power intensity, frequency, and time) on the stability and microscopic morphology of the nanocomposites were studied. Sonication improved the dispersion of CNFs into the polymer matrix, thereby improving the physical morphology, crystallinity, and thermomechanical properties of the nanocomposites. For example, compositing the polymer with $10 \% \mathrm{w} / \mathrm{w} \mathrm{CNF}$ resulted in $66 \%$ increase in crystallite size, $46 \%$ increase in micromolecular elastic strain, and $17 \%$ increase in lattice strain. Nevertheless, polymer degradation was observed following the ultrasound exposure. The constructed bionanocomposite could potentially be applied for organic electroconductive materials, biosensors and stimuli-responsive drug delivery devices.
\end{abstract}

\section{Introduction}

Increasing environmental pollution and degradation as a result of the utilization of petrochemical derived plastics have intensified the search for commercially viable biodegradable polymers [1]. Among the biopolymers are the microbial produced polyhydroxyalkanoates (PHA), which have received much attention due to their biocompatibility, biodegradability, and diverse structural composition, imparting onto them physicomechanical properties close to those of polyvinyl chloride and polyethylene terephthalate [2]. These combinations of excellent physicochemical properties led to the increasing commercial exploitation of PHA in different niche applications spanning from biomedical, packaging, automotive, infrastructure, and aerospace to military $[3,4]$.

Despite their promising commercial potentials, PHAs such as those with monomeric composition of 3-hydroxybutyric acid were reported to exhibit brittleness, low heat distortion temperature, poor gas-barrier properties, limited processing malleability, and ductility $[5,6]$. This in turn reduces the attractiveness and durability of the materials based on their strength, shelf lifetime, replacement, and maintenance cost. PHA modification and functionalization are expected to extend its quality or durability during application [7].

One approach for improving the thermomechanical stability of the biodegradable polymer is reinforcing the polymer using organic or inorganic nanofillers thereby extending their applications in aggressive environments where the neat polymers may fail [8-12]. Carbon nanoparticles were reported to exhibit high Young's modulus and excellent electrical and thermal conductivities [13, 14]. Introduction of carbon fullerene into polymer as nanocomposites helped to improve the material's physicomechanical properties such as polymer crystallization, gas-barrier property, thermomechanical strength, and electroconductivity surpassing those of the native biopolymers $[14,15]$. Hence, PHA composites based on carbon nanoparticles filler have attracted much interest and 
are considered industrially important in such applications like microelectronics, membrane separation, fire-resistance fabrics, petrochemical specialized filters, tissue scaffolding, and drug delivery devices.

The physicomechanical properties of the composited material are highly dependent upon the degree of nanofibers dispersion in the organic polymer matrix. Heterogeneous dispersion of nanofillers into the polymer matrix resulted in weak structural stability of the composite [16]. Insonation [17, 18], magnetron sputtering [19], spark plasma sintering [20], laser pyrolysis [21], and laser evaporation [22], and so forth, were employed to enhance the dispersion. In addition, surface functionalization was also reported to be an important way of improving the dispersion of nanoparticles into the polymer matrix [23-25]. While local turbulence due to the implosion of microbubbles from acoustic cavitation helps to disperse the carbon nanoparticles, the same high shear force could result in polymer degradation, thus affecting the thermomechanical properties of the fabricated material. Investigation on the thermomechanical stability of the fabricated polymer composite in relation to carbon nanoparticle loading and insonation variables is pertinent to the efficient process design and bionanocomposite properties.

In this study, the effects of carbon nanofibers (CNFs) loading and ultrasound irradiation variables on the physical morphology and thermomechanical stability of the poly3-hydroxyalkanoates nanocomposites were studied. While there have been reports on the fabrication of PHA/carbon nanoparticles composites [13, 14, 26-29], the combined effects as investigated in this study on the properties of PHA/CNFs nanocomposite have yet to be reported.

\section{Materials and Methods}

2.1. Materials. Carbon nanofibers (CNFs) of optical $\varnothing 200-$ $600 \mathrm{~nm}$ were purchased from US Research Nanomaterials, Inc. Houston, USA (CAS number 99685-96-8). All analytical grades chemicals used in the study were purchased from Merck Millipore (Darmstadt, Germany).

Biodegradable medium-chain-length PHA with comonomeric composition of $\mathrm{C}_{4: 0}$ to $\mathrm{C}_{10: 0}$ and an average molecular weight $(\mathrm{Mw})$ of $77.6 \mathrm{KDa}$ was obtained from reported fermentation culture conditions [1], using Delftia tsuruhatensis Bet002 as a producer microorganism and oleic acid as a sole carbon and energy source.

\subsection{Methods}

2.2.1. Oxidation of CNF. The polymer nanocomposite fabrication was performed according to the method reported previously $[29,30]$, with slight modification. About $0.5 \mathrm{~g}$ of CNF was mixed in $20 \mathrm{~mL}$ of piranha solution containing $4: 1 \mathrm{vol} / \mathrm{vol}$ of $97 \%$ sulfuric acid and $30 \%$ hydrogen peroxide and vortexed thoroughly. Then, the CNF mixture was oxidized by insonation at $40 \mathrm{kHz}, 11.5 \times 10^{3} \mathrm{~W} \mathrm{~m}^{-2}, 70^{\circ} \mathrm{C}$ for $1 \mathrm{~h}$ in a Multifrequency Ultrasonic Bath SB-300DTY (Ningbo Scientz Biotechnology Co., Ltd.; Zhejiang-China). The mixture was subsequently centrifuged at $9000 \times \mathrm{g}$ to recover the oxidized CNF, which was followed by sequential washing with distilled water until the $\mathrm{pH}$ of the drained water was approximately neutral. The oxidized CNFs pellet was then dried in a vacuum oven at $50^{\circ} \mathrm{C}$ for $24 \mathrm{~h}$ in the presence of phosphoric anhydride and stored there prior to composite fabrication.

2.2.2. Polymer Composite Fabrication. The dried oxidized CNFs were then composited with PHA via solution dispersion method. A varied amount of oxidized CNFs powder based on percentage polymer weight (w/w) was suspended in $20 \mathrm{~mL}$ dichloromethane solution containing $100 \pm$ $0.5 \mathrm{mg}$ mcl-PHA in a capped vial and sonicated using DTY ultrasonic Bath (SB-300DTY) at $40 \mathrm{kHz}, 4.7 \times 10^{3} \mathrm{~W} \mathrm{~m}^{-2}$, $50^{\circ} \mathrm{C}$ for 30 minutes. The solution was concentrated in rotary evaporator under reduced pressure to aliquot volume of about $2 \mathrm{~mL}$ and then casted in Petri dish into a thin film that was dried and kept in vacuo prior to further analyses. All reactions were carried out as described unless stated otherwise.

\subsubsection{Characterization of Polymer Composite}

(1) Field Emission Scanning Electron Microscopy (FESEM). The morphological characteristics of both the neat and the composited polymers were viewed in a high-resolution field emission scanning electron microscope (Quanta FEG 450) from FEI (Oregon, USA). The microscope was operated at high vacuum mode with an electron acceleration voltage of $5 \mathrm{kV}$ and a working distance of about $10 \mathrm{~mm}$. Thin films of neat polymer/polymer composite were mounted on brass stubs using double-sided cello tape and introduced into the viewing chamber of the instrument.

(2) FTIR-ATR Spectroscopy. A nondestructive attenuated total reflectance Fourier transform infrared spectra of the standard references and the composite polymer were recorded on Perkin-Elmer Spectrum 400 FT-IR and FT-NIR Spectrometer (Perkin-Elmer Inc., Wellesley, MA, USA) equipped with PIKE GladiATR hovering monolithic diamond ATR accessory (Pike technologies Inc., USA). Standard samples and their composites were placed on the monolithic diamond ATR probe and clamped against the diamond crystal plate using the force adapter. Thereafter, the samples were scanned over a range of $4000-400 \mathrm{~cm}^{-1}$ at $25^{\circ} \mathrm{C}$ as described previously [31].

(3) Wide Angle X-Ray Diffraction (WAXD) Analysis. The crystallite properties of both the pristine standards and the polymer composites were evaluated based on the diffractograms that were recorded on PANalytical Empyrean multipurpose X-ray diffractometer (PANalytical B.V.; The Netherlands) equipped with PIXcel ${ }^{3 \mathrm{D}} \mathrm{X}$-ray detector and anodic copper $\mathrm{K} \alpha_{1}$ radiation source $(\lambda=1.542 \AA)$ operated at $40 \mathrm{kV}$. Samples were scanned at $25^{\circ} \mathrm{C}$ over a range of $5^{\circ} \leq 2 \theta \leq$ $70^{\circ}$ with a step size of $0.026^{\circ} \mathrm{s}^{-1}$. The collected data of the diffraction pattern were analyzed using HighScore Plus version 3.0d software. 
(4) Simultaneous Thermal Analysis. The thermal stability of the neat polymer and its composite was evaluated by simultaneous thermal analysis (STA) using Perkin Elmer STA 6000 machine (Perkin-Elmer Inc., Wellesley, MA, USA) operated on tandem differential scanning calorimetry (DSC) and thermogravimetric analysis (TGA) at nitrogen flow rate of $20 \mathrm{~mL} \mathrm{~min}^{-1}$. Samples $(8 \mathrm{mg})$ were compressed in an aluminum boat and heated from $30^{\circ} \mathrm{C}$ to $550^{\circ} \mathrm{C}$ at a heating

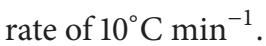

2.2.4. Viscosity Measurement. Viscosity of the polymer solution and that of the solvent was measured using a Sinewave Vibro SV-10 viscometer (A and D Company, Japan), as previously reported [31].

2.2.5. Numerical Calculations. The ultrasonic power was calculated using calorimetric analysis described in (1) as previously reported [32],

$$
P=\frac{m \times C_{p} \times \Delta T}{\Delta t},
$$

where $P$ is power (W), $m$ is the mass of the water $(\mathrm{g}), C_{p}$ is the specific heat capacity of water $\left(4.18 \mathrm{~J} \mathrm{~g}^{-1} \mathrm{~K}^{-1}\right), T$ is the temperature $(\mathrm{K})$, and $t$ is the insonation time $(\mathrm{s})$.

The calculated power is related to ultrasonic power intensity $I\left(\mathrm{~W} \mathrm{~m}^{-2}\right)$ as in

$$
I=\frac{P}{A}
$$

where $A$ represents the volumetric area $\left(\mathrm{m}^{2}\right)$.

The ultrasonic degradation rate $k\left(\mathrm{~s}^{-1}\right)$ is given by

$$
k=\frac{\left(M_{0}-M_{t}\right) / M_{0}}{\Delta t},
$$

where $M_{0}$ is the initial polymer molecular weight and $M_{t}$ is polymer molecular weight at time $t$.

The polymer molecular weight was determined using Mark-Houwink-Sakurada equation using the viscosity data as reported in literatures $[33,34]$.

The relative viscosity $\left(\eta_{r}\right)$ and specific viscosity $\left(\eta_{\mathrm{sp}}\right)$ are described by

$$
\begin{gathered}
\eta_{r}=\frac{\eta_{P}}{\eta_{s}}, \\
\eta_{\text {sp }}=1-\frac{1}{\eta_{r}},
\end{gathered}
$$

where $\eta_{p}$ and $\eta_{s}$ are the viscosities of polymer solution and the pure solvent, respectively.

The inherent viscosity $\left(\eta_{i}\right)$ is described by

$$
\eta_{i}=\frac{\ln \eta_{r}}{[P]},
$$

where $[P]$ is the polymer mass concentration in $\mathrm{g} \mathrm{L}^{-1}$.
Dividing the specific viscosity with respective polymer mass concentration gives the reduced (intrinsic) viscosity $\eta_{\text {red }}$ $\left(\mathrm{Lg}^{-1}\right)$ as shown in

$$
\eta_{\text {red }}=\frac{\eta_{\text {sp }}}{[P]}
$$

Using Huggins' empirical equations (see (7)), both the $\eta_{\text {red }}$ and $\eta_{i}$ were expressed as

$$
\begin{aligned}
\eta_{\text {red }} & =k^{\prime}[\eta]^{2}[P]+[\eta], \\
\eta_{i} & =k^{\prime \prime}[\eta]^{2}[P]+[\eta],
\end{aligned}
$$

where the constants $k^{\prime}$ and $k^{\prime \prime}$ were known to be a function of polymer, solvent, and temperature of the system; while $[\eta]$ is a hypothetical viscosity at zero polymer mass concentration.

From (7), a plot of $\eta_{\text {red }}$ versus $[P]$ and that of $\eta_{i}$ versus $[P]$ yield a slope of $k^{\prime}[\eta]^{2}$ and $k^{\prime \prime}[\eta]^{2}$, respectively, with an intercept of $[\eta]$ as depicted in Figure 1. Thus, from the plots, $[\eta]$ value could be considered to be a true value, if the observed $[\eta]$ from both Figures $1(\mathrm{a})$ and 1(b) are the same or $k^{\prime}-k^{\prime \prime}=0.5$.

Hence, the polymer viscosity molecular weight was determined according to semiempirical Mark-Houwink model (see (8)),

$$
[\eta]=K\left[\bar{M}_{\mathrm{vis}}\right]^{\alpha}
$$

where $K$ and $\alpha$ are Mark-Houwink empirical constants. For universal PHA, these constants were empirically determined by Nerkar et al. [34] to be $1.66 \times 10^{-4}$ and 0.689 , respectively.

$\mathrm{X}$-ray diffraction (XRD) pattern could provide an insight not only on the crystallite size and structure, but also on the internal strain-stress properties [35-38]. Hence, from the obtained XRD data, the crystallite size, internal elastic strains, and the percentage lattice strain were calculated using HighScore Plus ver 3.0d software and Williamson-Hall (see (10)) empirical equations [36-38]. Consider

$$
\begin{aligned}
D & =\frac{K \lambda}{\beta \cos \theta}, \\
\beta \cos \theta & =\frac{K \lambda}{D}+4 \varepsilon \sin \theta,
\end{aligned}
$$

where $D$ is the crystallite size, $K$ is Debye-Scherrer's shape factor constant denoted by 0.9 [36-38], $\lambda$ is the $\mathrm{Cuk}_{\alpha}$ radiation wave length, and $\beta$ is the full width at half maximum (FWHM) intensity (referring to the peak-width measured at position halfway from the peak's apex, roughly donated by the difference between the $2 \theta$ base line points of the peak as highlighted by Cullity [36]).

Thus, from (10), a plot of $\beta \cos \theta$ as a function of $4 \sin \theta$ gives a slope of the internal elastic strain $(\varepsilon)$ as reported [38, 39].

Polymer thermal degree of crystallinity $\left(X_{c}\right)$ is calculated according to (11) as described in literatures [27, 40]. Consider

$$
X_{c}=\frac{\Delta H_{m}}{\left(m_{p} \cdot \Delta H_{m}^{\circ}\right)},
$$




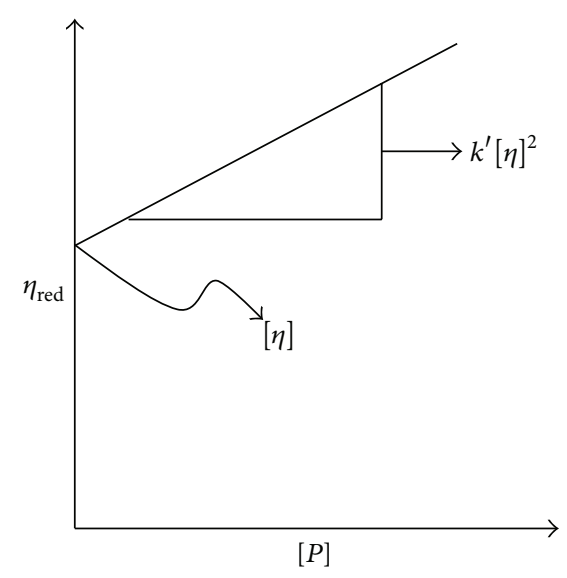

(a)

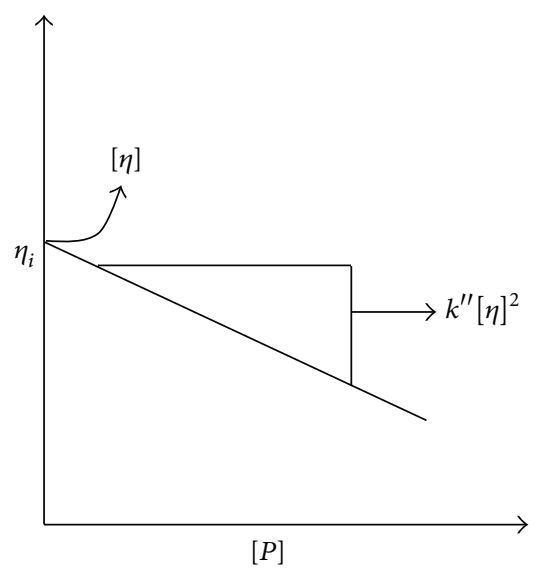

(b)

FIGURE 1: Linearized plots of Huggins relationships using (a) reduced viscosity and (b) inherent viscosity as a function of polymer mass concentration.

where $\Delta H_{m}$ is the STA melting enthalpy of the PHA, $\Delta H_{m}^{\circ}$ is the melting enthalpy of the standard crystalline PHBV (146.6 $\left.\mathrm{Jg}^{-1}\right)$, and $m_{p}$ is the PHA mass fraction in the composite.

\section{Results and Discussion}

3.1. Characterization of Polymer Nanocomposite. The FESEM micrograph of the polymer composites showed the dispersed carbon nanotubes within the polymer matrix (Figure 2). It can be observed that in the ultrasound-assisted PHA/CNF fabrication, acoustic generated turbulence resulted in an exfoliated morphological characteristic in the polymer composite for both lower (Figure 2(a)) and higher (Figure 2(b)) CNF loadings. This indicated well-dispersed CNTs within the PHA matrix. On the other hand, conventional meltmixed PHA/CNF composite was observed to produce similar exfoliated morphology for composite at lower CNF loading $(0.5 \%)$ (Figure $2(\mathrm{c})$ ). At higher CNF loading (5\%), tactoid aggregation morphology was observed in the composite prepared via solvent dispersion route (Figure 2(d)).

The oxidation of the carbon nanofibers and successful dispersion of CNTs within the polymer matrices were indicated by the FTIR-ATR spectra as shown in Figure 3. The observed prominent FTIR absorption at $3394 \mathrm{~cm}^{-1}$ in the oxidized CNF sample signified the presence of hydroxyl ($\mathrm{OH}$ ) group. This band was observed to be highly reduced in composite polymer sample, indicating successful dispersion of the CNF within the polymer matrix. The absorption was also found to be less prominent in both the pristine CNF and neat PHA samples.

Absorption at 2925 and $2845 \mathrm{~cm}^{-1}$ was attributed to both asymmetric $\mathrm{CH}_{3}$ and symmetric $\mathrm{CH}_{2}$ vibrations in the samples, respectively. The presence of carbonyl ester bond in neat PHA sample was assigned to the absorption at $1735 \mathrm{~cm}^{-1}$. This vibration was shifted to $1727 \mathrm{~cm}^{-1}$ in polymer-CNF composite sample signifying an increase in crystallinity. This observation was found to be in agreement with reported shifting of the said band in polyester carbonyl groups due to the increase in crystallinity [41]. The absorption band at $1633 \mathrm{~cm}^{-1}$ belongs to $\mathrm{C}=\mathrm{C}[42,43]$. The series of bands between 1543 and $1450 \mathrm{~cm}^{-1}$ were reported to be due to $\mathrm{CH}$ bending of methyl and methylene groups [41]; this region is also known to overlap with $\mathrm{C}-\mathrm{O}-\mathrm{C}$ stretching vibrations $[43,44]$. Furthermore, in the pristine CNF samples, the sharp absorptions at 1545 and about $823 \mathrm{~cm}^{-1}$ illustrated the reported normal vibration of carbon nanoparticles phonon modes $\mathrm{E}_{1 \mathrm{u}}$ and $\mathrm{A}_{2 \mathrm{u}}$, respectively $[43,45]$. In the oxidized $\mathrm{CNF}$, the absorption at $1367 \mathrm{~cm}^{-1}$ is due to the $\mathrm{C}-\mathrm{OH}$ stretching vibrations [42], whereas series of absorptions in all spectra at $1276-736 \mathrm{~cm}^{-1}$ was reported to be due to $\mathrm{C}-\mathrm{O}$ stretching vibrations $[42,43]$. In fact, the relative intensity and partial separation of bands at $1250-950 \mathrm{~cm}^{-1}$ were suggested to be dependent of the amounts of $\mathrm{OH}$ deformation and $\mathrm{C}-\mathrm{O}$ stretching combination in surface of phenolic rings and aromatic carboxylic acids [43]. In agreement with this observation, the CNF oxidation was strongly indicated in this study due to the presence of sharp and highly intense absorption at $1056 \mathrm{~cm}^{-1}$ wavelength. This band was observed to be less prominent in pristine CNF sample, whereas the intensity of the peak was significantly reduced in the polymer composite sample probably due to dispersion effect.

The crystalline nature of the tested samples is shown in Figure 4. In the CNF diffractogram, an intense crystalline peak was observed at $2 \theta$ value of $27.4^{\circ}$, a typical characteristics of graphite hexagonal peak at (002) plane [42, 45]. The $d$ spacing of this peak was found to stand at $0.33 \mathrm{~nm}$ with corresponding percentage lattice strain of $2.6 \%$ and micromolecular elastic strain of 0.3 . Similar $d$-spacing value based on peak 002 reflection was observed by Vinayan et al. [42] in multiwalled carbon nanotube $(0.336 \mathrm{~nm})$ and graphite sheets $(0.334 \mathrm{~nm})$. For oxidized CNF, this intense crystalline peak (002) was found to be shifted to lower values of $2 \theta\left(25.9^{\circ}\right)$ with corresponding increase in the $d$-spacing to $0.347 \mathrm{~nm}$ and lattice strain $3.88 \%$ and elastic strain of 0.46 with an appearance of broader peak at $43.8^{\circ}$ (Figure 4 ). The decrease in the $2 \theta$ angle and the corresponding increase in interfiber $d$ spacing were suggested to be due to the dispersion of oxygen containing functional groups, which could also caused the 


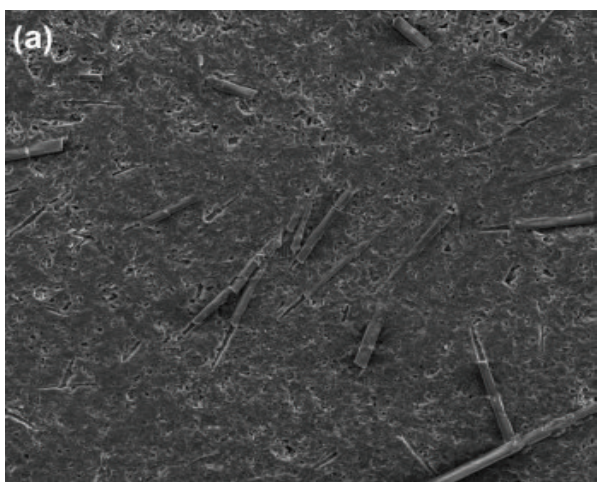

(a)

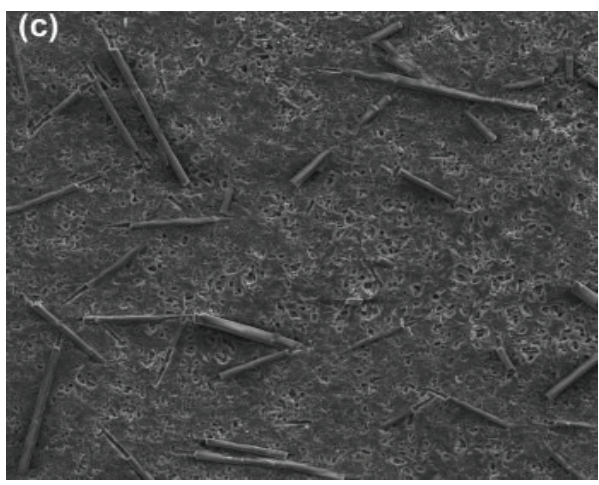

(c)

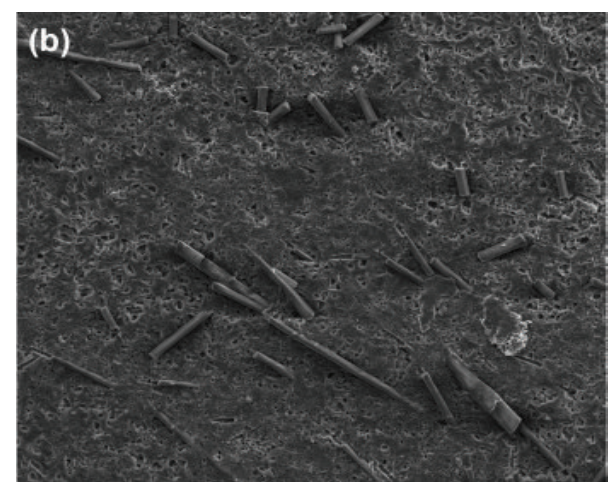

(b)

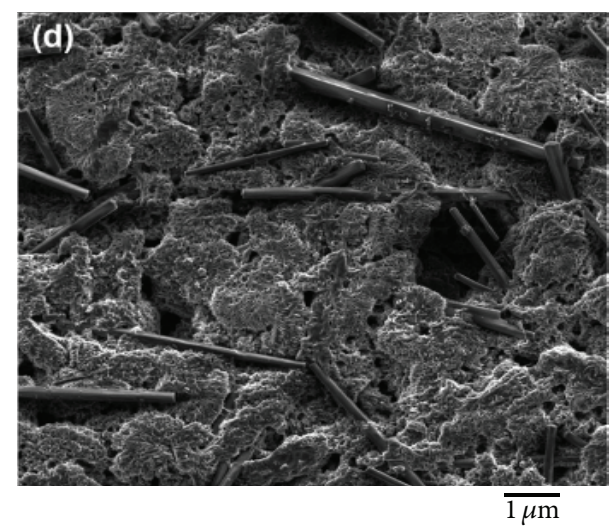

(d)

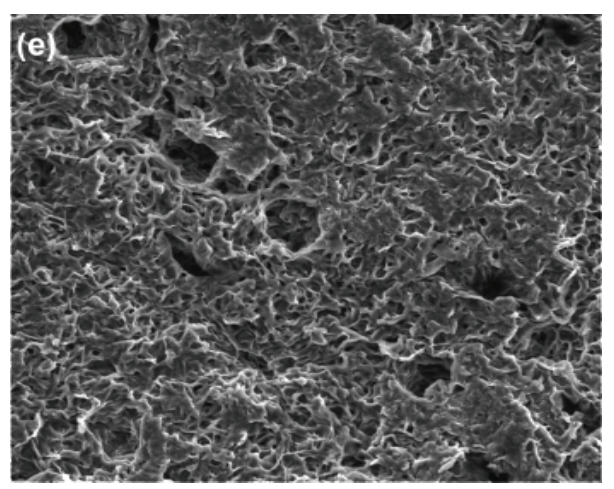

(e)

FIGURE 2: Field emission scanning electron micrographs of PHA composites and neat PHA; (a) ultrasound-assisted PHA composite at 0.5\% CNF, (b) ultrasound-assisted PHA composite at 5\% CNF, (c) normal solution dispersion PHA composite at 0.5\% CNF, (d) normal solution dispersion PHA composite at 10\% CNF, and (e) neat PHA. All micrographs were recorded at 5000x magnification.

observed increase in elastic and lattice strain. The observed broadening of the peak 101 reflection was previously linked to the lattice distortion along the $c$-axis [42].

In comparison to the pristine CNF, the standard PHA was observed to display the typical polymeric crystallite reflections at (020), (110), (111), and (040) planes (Figure 4). Additionally, the 040 reflection peak in the neat PHA was observed to be broader due to the presence of less perfect crystal structure in the neat PHA. Intercalating the oxidized CNF with PHA resulted in the intense appearance of 002 reflection at $27.5^{\circ}$ on $2 \theta$ scale. In contrast to pristine CNF, the peak in this composite was observed to exhibit a decrease in the $d$-spacing to $0.32 \mathrm{~nm}$ indicating utilization of oxygen functional groups to form polymer-CNF dispersion. In addition, the 002 reflection peak was found to be less intense in PHA/CNF composite difractogram as compared to the observed 002 peak in the pristine CNF. This was attributed to the strong hydrogen bonding between the carboxyl groups of the organic polymer matrix and the hydroxyl groups of the CNF [40].

It is noted that compositing the PHA with $\mathrm{CNF}$ at different fractions increased the PHA crystallinity due to the 


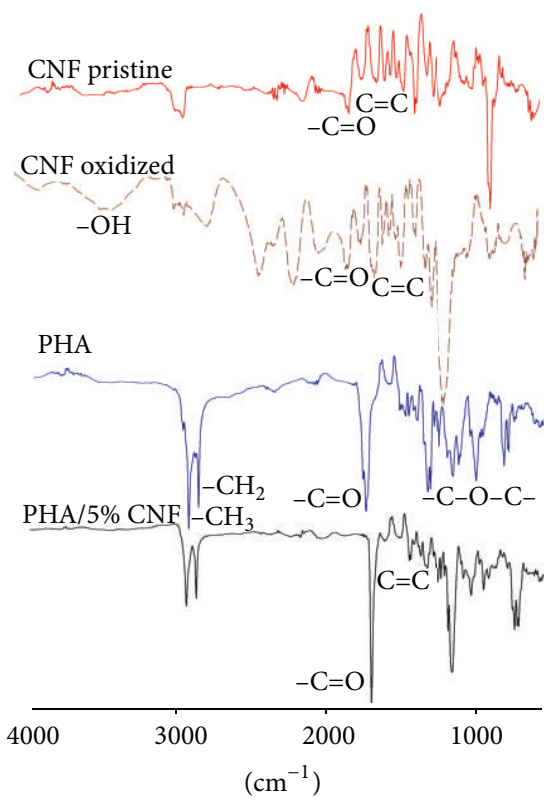

FIGURE 3: FTIR-ATR spectra of the standards and polymer composites.

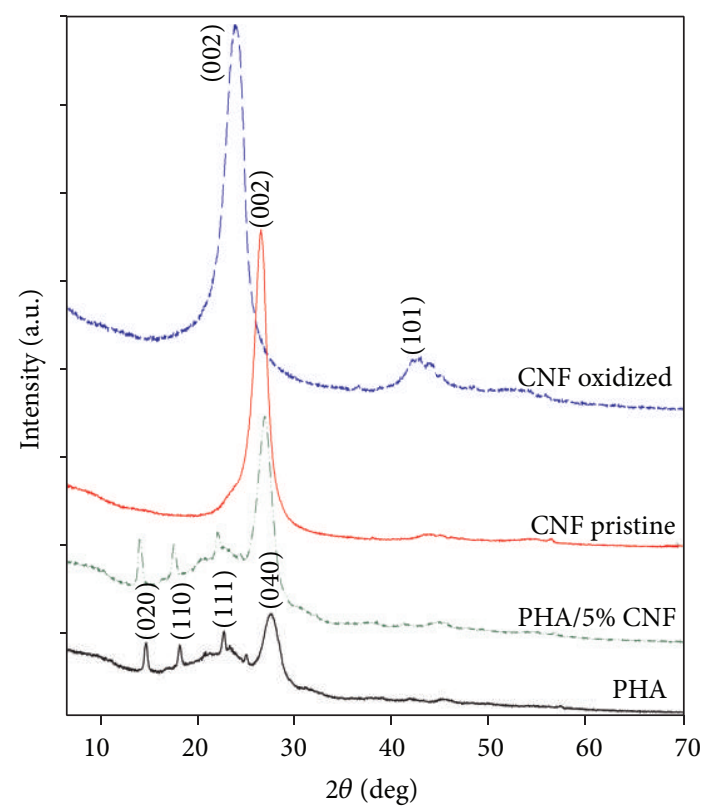

FIgURE 4: X-ray diffractogram of the PHA/CNF composite and the standards.

observed increase in crystallite size, micromolecular elastic strain, and percentage lattice strain. For example, in Table 1, the crystallite size at 020 reflection was observed to be increased from $4.91 \mathrm{~nm}$ in neat PHA to $5.15 \mathrm{~nm}$ in PHA composite containing $0.5 \%$ CNF. It has been documented that molecular crystallization is highly dependent on the rate of nucleation and crystal growth rate [27]. The observed elevated crystallite size and the increase in peak intensity (Figure 4) strongly indicated the crystallization effect of CNF on PHA. Similar observations were reported on PHBV composites a [40, 46]. Shan et al. [27] reported that the addition of carbon nanotubes greatly enhances the nucleation rate of PHBV resulting in efficient crystallization. Similarly, addition of multiwalled carbon nanotubes also resulted in increased crystallization in PHBV [47].

To substantiate the hypothesis, the crystallite size was observed to keep increasing with the increase in CNF content up to $7 \%(14.43 \mathrm{~nm})$ after which further addition resulted in poor crystallization (Table 1). In fact, compositing the neat PHA with $10 \%$ oxidized CNF resulted in a lower crystallite size $(14.22 \mathrm{~nm})$ with corresponding decrease in elastic strain (2.53) and percentage lattice strain (2.62\%). This could be due to the reported interference in the macromolecular continuity of the polymer such as that observed in polyethylene [48] and PHBV [27] matrices caused by higher loading of carbon nanotubes in polymer composite. This affects the mobility of the polymer chains resulting in gain boundaries and crystallite distortion.

The observed increase in polymer crystallinity as a result of CNTs composition was corroborated by the data obtained from simulteneous thermal analysis (Table 1). From the STA thermograms, the degree of crystallinity was calculated according to empirical model given in (11). In accordance with observed increased in crystallite size, the thermal stability of the polymer composite based on melting temperature $\left(T_{m}\right)$ and degradation temperature $\left(T_{d}\right)$ as well as the degree of crystallinity were all found to be increased upon dispersion of the CNF into the organic polymer matrix in a CNF fraction dependent fashion (Table 1). Compositing the neat PHA with $7 \%(\mathrm{w} / \mathrm{w})$ CNF was found to elevate the crystallinity by about $58.2 \%$ resulting in corresponding increase of $T_{m}$ and $T_{d}$ from $163.6^{\circ} \mathrm{C}$ and $272.2^{\circ} \mathrm{C}$ to $183.5^{\circ} \mathrm{C}$ and $331.2^{\circ} \mathrm{C}$, respectively. In agreement with earlier WAXD observation, polymer composited samples of higher CNF loading (10\% $\mathrm{w} / \mathrm{w})$ were observed to exhibit lower $T_{m}$ and $T_{d}$. This could probably be attributed to the crystallite distortion as a result of higher CNF loading. Thus, it could suffice to say that in this study, at higher CNF loading incurs similar effect, which resulted in the observed decrease in the crystallite size and crystallinity.

3.2. Effects of Insonation Variables (Power Intensity, Frequency, and Time) on the PHA/CNF Nanocomposite Thermophysical Properties and Stability. The effects of sonication intensity on the dispersion of $0.5 \%$ CNTs $(\mathrm{w} / \mathrm{w})$ were studied over a range of $1.1-7.5 \times 10^{3} \mathrm{~W} \mathrm{~m}^{-2}$ at $40 \mathrm{KHz}, 50^{\circ} \mathrm{C}$ for 30 minutes. At power intensity lower than $4.7 \times 10^{3} \mathrm{~W} \mathrm{~m}^{-2}$, the tactoid aggregated morphology was clearly evident (Figures 5(a) and 5(b)). The characteristic exfoliated morphology of PHA-CNF composite was observed at $4.7 \times 10^{3}$ and $7.5 \times 10^{3} \mathrm{~W} \mathrm{~m}^{-2}$ power intensities (Figure 5), indicative of well-dispersed CNTs. However, at higher intensities such as $7.5 \times 10^{3} \mathrm{~W} \mathrm{~m}^{-2}$ reduction in polymer molecular weight was observed possibly due to partial degradation of the polymer as a result of high turbulent generated by cavitation implode (Table 2). This could explain the observation made in the sample of nanocomposite irradiated at higher power intensity (Figure 5(d), inset) where a segment appeared to 


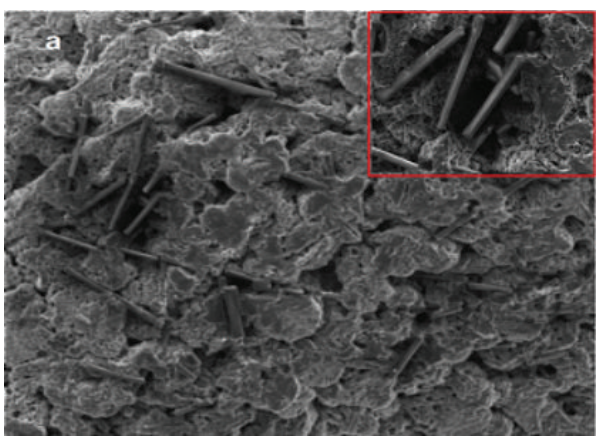

$1 \mu \mathrm{m}$

(a)

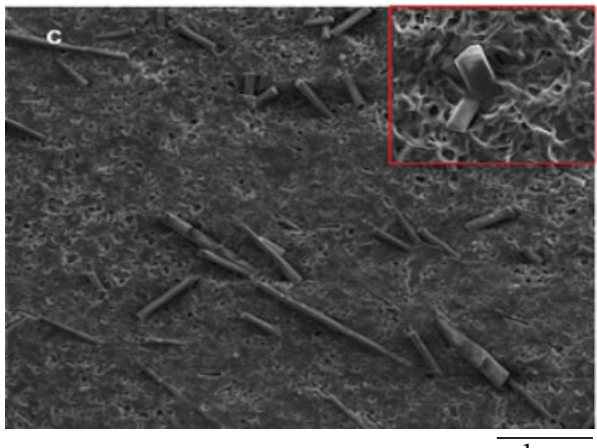

$1 \mu \mathrm{m}$

(c)

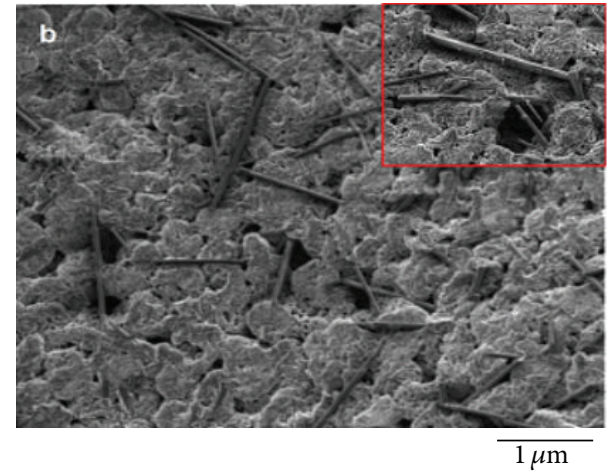

(b)

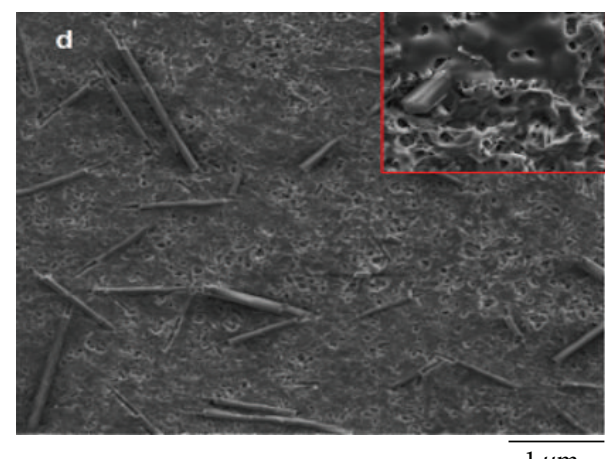

(d)

FiguRE 5: FESEM micrographs displaying the effect of sonication power intensity (I) on CNF dispersion (a) $1.1 \times 10^{3} \mathrm{~W} \mathrm{~m}{ }^{-2}$, (b) $2.1 \times$ $10^{3} \mathrm{~W} \mathrm{~m}^{-2}$, (c) $4.7 \times 10^{3} \mathrm{~W} \mathrm{~m}^{-2}$, and (d) $7.5 \times 10^{3} \mathrm{~W} \mathrm{~m}^{-2}$ (all micrographs were recorded at 5000x magnification). The insets are micrographs of the samples at 10000x magnification; the observed polymer melting (sample (d), inset) could be due to lower $M_{v}$ of polymer matrix due to partial degradation by high sonication intensity.

TABLE 1: STA crystallinity and WAXD crystallite size data of neat PHA and its CNF composites.

\begin{tabular}{lccccccc}
\hline Sample & $T_{m}\left({ }^{\circ} \mathrm{C}\right)$ & $\Delta H_{m}\left(\mathrm{Jg}^{-1}\right)$ & $T_{d}\left({ }^{\circ} \mathrm{C}\right)$ & $X_{c}(\%)$ & $D_{020,040}(\mathrm{~nm})$ & $\varepsilon$ & Lattice strain $(\%)$ \\
\hline PHA & 163.6 & 53.2 & 272.2 & 36.3 & 4.91 & 5.15 & 1.42 \\
PHA/0.5\% CNF & 169.4 & 67.8 & 289.5 & 46.5 & 7.58 & 3.10 \\
PHA/5\% CNF & 178.7 & 88.1 & 326.7 & 73.3 & 8.46 & 2.81 & 3.61 \\
PHA/7\% CNF & 183.5 & 97.4 & 331.2 & 86.8 & 14.43 & 2.62 & 3.74 \\
PHA/10\% CNF & 202.7 & 92.6 & 325.6 & 86.2 & 14.22 & 2.53 \\
\hline
\end{tabular}

have melted due to the heating effect by the applied electron voltage of FESEM. The partial degradation of the polymer caused by high insonation intensity could be responsible in lowering the melting temperature of PHA at the particular segment. Similar melting effect, however, was not observed in the other samples of nanocomposite irradiated at lower power intensity (Figures 5(a)-5(c), insets). Notwithstanding the observed degradation, the improved CNF dispersion with increasing power intensity was observed to increase the polymer thermal stability and crystalline properties (Table 2 ). Increasing the power intensity from $1.1 \times 10^{3}$ to $7.5 \times$ $10^{3} \mathrm{~W} \mathrm{~m}^{-2}$ resulted in an observed decrease in $M_{v}$ from 75.2 to $59.8 \mathrm{kDa}$. The possible mechanism of the polymer degradation with increasing sonication power was reported previously [32].
TABLE 2: Effects of sonication power intensity on polymer composite.

\begin{tabular}{lcccc}
\hline$I\left(\times 10^{3} \mathrm{~W} \mathrm{~m}^{-2}\right)$ & $M_{v}(\mathrm{KDa})$ & $T_{m}\left({ }^{\circ} \mathrm{C}\right)$ & $T_{d}\left({ }^{\circ} \mathrm{C}\right)$ & $X_{c}(\%)$ \\
\hline 1.1 & 75.2 & 132.2 & 298.4 & 71.2 \\
2.1 & 72.9 & 144.2 & 316.9 & 72.3 \\
4.7 & 66.7 & 156.7 & 320.6 & 76.8 \\
7.5 & 59.8 & 160.1 & 326.4 & 85.4 \\
\hline
\end{tabular}

Specifically, the increase in sonication power intensity from $1.1-7.5 \times 10^{3} \mathrm{~W} \mathrm{~m}^{-2}$ resulted in increased dispersion of the CNFs incurring the corresponding increase in $T_{m}$, $T_{d}$, and $X_{c}$ from $132.2^{\circ} \mathrm{C}, 298.4^{\circ} \mathrm{C}$, and $71.2 \%$ to $160.1^{\circ} \mathrm{C}$, $326.4^{\circ} \mathrm{C}$, and $85.4 \%$, respectively (Table 2 ). Based on the trend of increase in the $T_{m}$ and $T_{d}$ with power intensity (Table 2), 


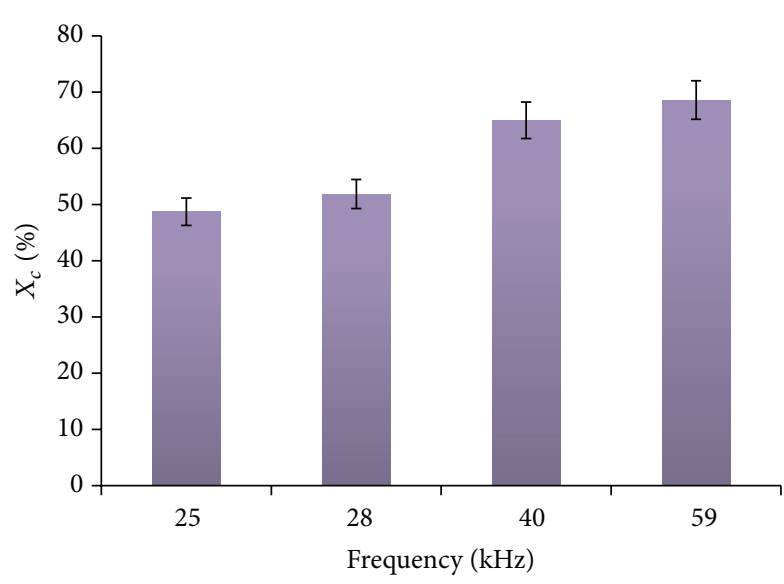

(a)

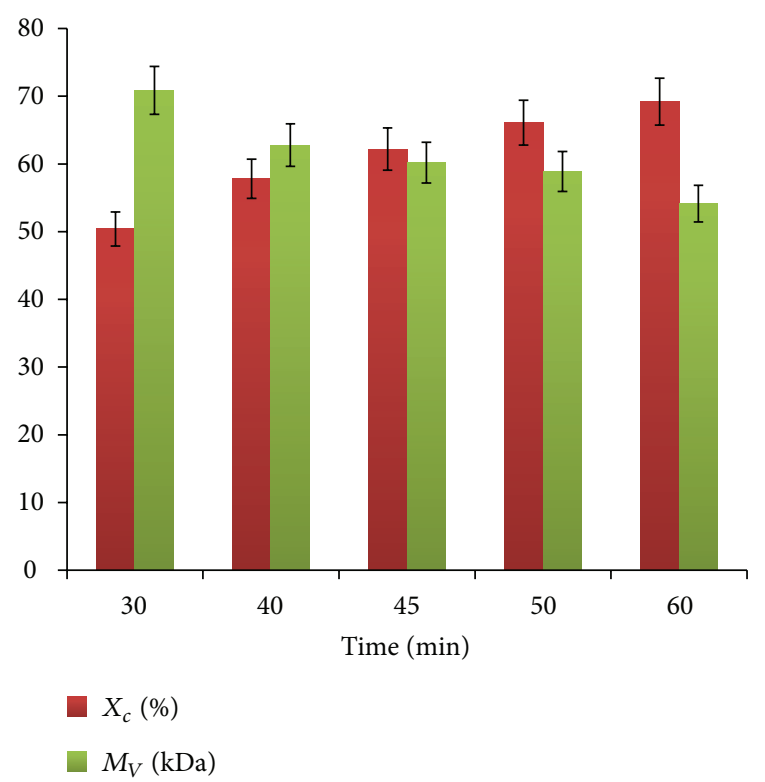

(b)

Figure 6: Effects of sonication frequency $\left(30 \mathrm{~min}, 50^{\circ} \mathrm{C}\right)$ and time $\left(40 \mathrm{kHz}, 50^{\circ} \mathrm{C}\right)$ on the crystallinity $\left(X_{c}\right)$ and weight average molecular weight $\left(M_{w}\right)$ of polymer composite.

it was expected that the increase in $T_{m}$ and $T_{d}$ at $7.5 \times$ $10^{3} \mathrm{~W} \mathrm{~m}^{-2}$ would be significantly higher than the recorded values. However, this was not the case. As the observation was attributed to the partial polymer degradation resulting in lower $M_{v}$, and, hence, small increase in the $T_{m}$ and $T_{d}$ at $7.5 \times 10^{3} \mathrm{~W} \mathrm{~m}^{-2}$.

When the insonation frequency was varied from 59 to $25 \mathrm{KHz}$ for 30 minutes irradiation period, reduction in the crystallinity of the polymer composite was observed (Figure 6(a)). This was attributed to the increase in polymer degradation (hence control crystallinity) with a decrease in applied ultrasonic frequency. There is a wealth of experimental information that showed increased polymer degradation with a decrease in insonation frequency $[49,50]$. At $40 \mathrm{kHz}$ sonication frequency, negligible decrease and increase in the viscosity average molecular weight $\left(M_{w}\right)$, and crystallinity $\left(X_{c}\right)$ were observed, respectively, when irradiation time was prolonged from 30 minutes to 40 minutes. After 40 minutes and above of acoustic irradiation, no significant decrease nor increase in the weight average molecular weight $\left(M_{w}\right)$ and crystallinity $\left(X_{c}\right)$ was observed, respectively (Figure 6(b)). This indicated that irradiation time could be limited to 30 minutes.

\section{Conclusions}

The application of sonication enhanced the dispersion of CNFs into the polymer matrix, thereby improving the physical morphology, crystallinity, and thermomechanical properties of the nanocomposites. Controlling the sonication variables such as power intensity, frequency, and time as well as the fraction of CNFs loading could result in efficient fabrication of smart and biocompatible nanocomposites that could find applications in organic electroconductive materials, biosensors, and drug delivery devices.

\section{Conflict of Interests}

The authors declare that there is no conflict of interests regarding the publication of this paper.

\section{Acknowledgment}

We acknowledged University of Malaya for providing the research Grants nos. BK014-2014, RG024-2012A, UM.C/625/ 1/HIR/MOHE/05, and PV036-2012A.

\section{References}

[1] A. M. Gumel, M. S. M. Annuar, and T. Heidelberg, "Effects of carbon substrates on biodegradable polymer composition and stability produced by Delftia tsuruhatensis Bet002 isolated from palm oil mill effluent," Polymer Degradation and Stability, vol. 97, no. 8, pp. 1224-1231, 2012.

[2] A. Poli, P. di Donato, G. R. Abbamondi, and B. Nicolaus, "Synthesis, production, and biotechnological applications of exopolysaccharides and polyhydroxyalkanoates by Archaea," Archaea, vol. 2011, Article ID 693253, 13 pages, 2011.

[3] A. M. Gumel, M. S. M. Annuar, and Y. Chisti, "Recent advances in the production, recovery and applications of polyhydroxyalkanoates," Journal of Polymers and the Environment, vol. 21, no. 2, pp. 580-605, 2013.

[4] J. Rhim, H. Park, and C. Ha, "Bio-nanocomposites for food packaging applications," Progress in Polymer Science, vol. 38, no. 10-11, pp. 1629-1652, 2013. 
[5] S. S. Ray and M. Bousmina, "Biodegradable polymers and their layered silicate nanocomposites: In greening the 21st century materials world," Progress in Materials Science, vol. 50, no. 8, pp. 962-1079, 2005.

[6] C. Johansson, "Bio-nanocomposites for food packaging applications," in Nanocomposites with Biodegradable Polymers: Synthesis, Properties and Future Perspectives, V. Mittal, Ed., pp. 348367, Oxford University Press, Oxford, UK, 2011.

[7] A. M. Gumel, M. S. M. Annuar, and T. Heidelberg, "Current application of controlled degradation processes in polymer modification and functionalization," Journal of Applied Polymer Science, vol. 129, no. 6, pp. 3079-3088, 2013.

[8] S. Ray and M. Bousmina, Biodegradable Polymer/Layered Silicate Nanocomposites, Woodhead Publishing, Cambridge, UK, 2006.

[9] R. Zhang, H. Huang, W. Yang, X. Xiao, and Y. Hu, "Preparation and characterization of bio-nanocomposites based on poly(3-hydroxybutyrate-co-4-hydroxybutyrate) and CoAl layered double hydroxide using melt intercalation," Composites Part A Applied Science and Manufacturing, vol. 43, no. 4, pp. 547-552, 2012.

[10] K. M. Dean, P. Sangwan, C. Way, and M. A. L. Nikolic, "Biodegradability characterization of polymer nanocomposites," Characterization Techniques for Polymer Nanocomposites, vol. 3, 2012.

[11] C. Zhijiang, Y. Guang, and J. Kim, "Biocompatible nanocomposites prepared by impregnating bacterial cellulose nanofibrils into poly(3-hydroxybutyrate)," Current Applied Physics, vol. 11, no. 2, pp. 247-249, 2011.

[12] A. Sionkowska, "Current research on the blends of natural and synthetic polymers as new biomaterials: review," Progress in Polymer Science, vol. 36, no. 9, pp. 1254-1276, 2011.

[13] S. K. Misra, F. Ohashi, S. P. Valappil et al., "Characterization of carbon nanotube (MWCNT) containing $\mathrm{P}(3 \mathrm{HB}) /$ bioactive glass composites for tissue engineering applications," Acta Biomaterialia, vol. 6, no. 3, pp. 735-742, 2010.

[14] S. I. Yun, V. Lo, J. Noorman et al., "Morphology of composite particles of single wall carbon nanotubes/biodegradable polyhydroxyalkanoates prepared by spray drying," Polymer Bulletin, vol. 64, no. 1, pp. 99-106, 2010.

[15] J. K. Pandey, K. Raghunatha Reddy, A. P. Kumar, and R. P. Singh, "An overview on the degradability of polymer nanocomposites," Polymer Degradation and Stability, vol. 88, no. 2, pp. 234-250, 2005.

[16] H. Ishida, S. Campbell, and J. Blackwell, "General approach to nanocomposite preparation," Chemistry of Materials, vol. 12, no. 5, pp. 1260-1267, 2000.

[17] E. C. Lee, D. F. Mielewski, and R. J. Baird, "Exfoliation and dispersion enhancement in polypropylene nanocomposites by in-situ melt phase ultrasonication," Polymer Engineering and Science, vol. 44, no. 9, pp. 1773-1782, 2004.

[18] C. Park, Z. Ounaies, K. A. Watson et al., "Dispersion of single wall carbon nanotubes by in situ polymerization under sonication," Chemical Physics Letters, vol. 364, no. 3-4, pp. 303308, 2002.

[19] J. Musil and P. Baroch, "High-rate pulsed reactive magnetron sputtering of oxide nanocomposite coatings," Vacuum, vol. 87, pp. 96-102, 2013.

[20] A. Nieto, D. Lahiri, and A. Agarwal, "Synthesis and properties of bulk graphene nanoplatelets consolidated by spark plasma sintering," Carbon, vol. 50, no. 11, pp. 4068-4077, 2012.
[21] Y. Leconte, S. Veintemillas-Verdaguer, M. P. Morales et al., "Continuous production of water dispersible carbon-iron nanocomposites by laser pyrolysis: application as MRI contrasts," Journal of Colloid and Interface Science, vol. 313, no. 2, pp. 511-518, 2007.

[22] R. A. McGill, D. B. Chrisey, and A. Pique, "Fabrication of conductive/non-conductive nanocomposites by laser evaporation," U. S. Patents, US 6660343 B2, 2003.

[23] Y. Qian, C. I. Lindsay, C. MacOsko, and A. Stein, "Synthesis and properties of vermiculite-reinforced polyurethane nanocomposites," ACS Applied Materials and Interfaces, vol. 3, no. 9, pp. 3709-3717, 2011.

[24] O. Morales-Teyssier, S. Sánchez-Valdes, and L. F. Ramos-de Valle, "Effect of carbon nanofiber functionalization on the dispersion and physical and mechanical properties of polystyrene nanocomposites," Macromolecular Materials and Engineering, vol. 291, no. 12, pp. 1547-1555, 2006.

[25] Y. Qian, W. Liu, Y. T. Park et al., "Modification with tertiary amine catalysts improves vermiculite dispersion in polyurethane via in situ intercalative polymerization," Polymer, vol. 53, no. 22, pp. 5060-5068, 2012.

[26] M. D. Sanchez-Garcia, J. M. Lagaron, and S. V. Hoa, "Effect of addition of carbon nanofibers and carbon nanotubes on properties of thermoplastic biopolymers," Composites Science and Technology, vol. 70, no. 7, pp. 1095-1105, 2010.

[27] G.-F. Shan, X. Gong, W.-P. Chen, L. Chen, and M.-F. Zhu, "Effect of multi-walled carbon nanotubes on crystallization behavior of poly(3-hydroxybutyrate-co-3-hydroxyvalerate)," Colloid and Polymer Science, vol. 289, no. 9, pp. 1005-1014, 2011.

[28] S. I. Yun, G. E. Gadd, B. A. Latella, V. Lo, R. A. Russell, and P. J. Holden, "Mechanical properties of biodegradable polyhydroxyalkanoates/single wall carbon nanotube nanocomposite films," Polymer Bulletin, vol. 61, no. 2, pp. 267-275, 2008.

[29] Y. T. Ong, A. L. Ahmad, S. H. S. Zein, K. Sudesh, and S. H. Tan, "Poly (3-hydroxybutyrate)-functionalised multi-walled carbon nanotubes/chitosan green nanocomposite membranes and their application in pervaporation," Separation and Purification Technology, vol. 76, no. 3, pp. 419-427, 2011.

[30] K. J. Ziegler, Z. Gu, H. Peng, E. L. Flor, R. H. Hauge, and R. E. Smalley, "Controlled oxidative cutting of single-walled carbon nanotubes," Journal of the American Chemical Society, vol. 127, no. 5, pp. 1541-1547, 2005.

[31] A. Gumel, M. Annuar, and Y. Chisti, "Ultrasound-assisted enzymatic synthesis of poly- $\varepsilon$-caprolactone: kinetic behavior and reactor design," International Journal of Chemical Reactor Engineering, vol. 11, no. 1, pp. 609-617, 2013.

[32] A. M. Gumel, M. S. M. Annuar, and Y. Chisti, "Lipase catalyzed ultrasonic synthesis of poly-4-hydroxybutyrate-co-6- hydroxyhexanoate," Ultrasonics Sonochemistry, vol. 20, no. 3, pp. 937947,2013

[33] S. Harding, "Intrinsic viscosity," in Encyclopedia of Biophysics, G. K. Roberts, Ed., pp. 1123-1129, Springer, Berlin, Germany, 2013.

[34] M. Nerkar, J. A. Ramsay, B. A. Ramsay, M. Kontopoulou, and R. A. Hutchinson, "Determination of mark-houwink parameters and absolute molecular weight of medium-chain-length poly(3Hydroxyalkanoates)," Journal of Polymers and the Environment, vol. 21, no. 1, pp. 24-29, 2013.

[35] N. Sasaki and S. Odajima, "Stress-strain curve and Young's modulus of a collagen molecule as determined by the X-ray diffraction technique," Journal of Biomechanics, vol. 29, no. 5, pp. 655-658, 1996. 
[36] B. D. Cullity, Elements of X-Ray Diffraction, Addison-Wesley, Reading, Mass, USA, 1969.

[37] K. Tan, M. R. Johan, R. Ahmad, N. A. Kadri, N. M. Zain, and A. K. Arof, "Mechanical analysis of the effects of different mechanical alloying periods on $\mathrm{Ni}_{3} \mathrm{Al}$ intermetallic compound fabrication quality," International Journal of Electrochemical Science, vol. 7, no. 4, pp. 3765-3772, 2012.

[38] V. D. Mote, Y. Purushotham, and B. N. Dole, "Williamson-Hall analysis in estimation of lattice strain in nanometer-sized $\mathrm{ZnO}$ particles," Journal of Theoretical and Applied Physics, vol. 6, no. 1, pp. 1-8, 2012.

[39] A. Khorsand Zak, W. H. Abd. Majid, M. E. Abrishami, and R. Yousefi, "X-ray analysis of $\mathrm{ZnO}$ nanoparticles by WilliamsonHall and size-strain plot methods," Solid State Sciences, vol. 13, no. 1, pp. 251-256, 2011.

[40] L. N. Carli, J. S. Crespo, and R. S. Mauler, "PHBV nanocomposites based on organomodified montmorillonite and halloysite: the effect of clay type on the morphology and thermal and mechanical properties," Composites A: Applied Science and Manufacturing, vol. 42, no. 11, pp. 1601-1608, 2011.

[41] S. Randriamanhefa, E. Renard, P. Guérin, and V. Langlois, "Fourier transform infrared spectroscopy for screening and quantifying production of PHAs by Pseudomonas grown on sodium octanoate," Biomacromolecules, vol. 4, no. 4, pp. 10921097, 2003.

[42] B. P. Vinayan, R. Nagar, V. Raman, N. Rajalakshmi, K. S. Dhathathreyan, and S. Ramaprabhu, "Synthesis of graphene-multiwalled carbon nanotubes hybrid nanostructure by strengthened electrostatic interaction and its lithium ion battery application," Journal of Materials Chemistry, vol. 22, no. 19, pp. 9949-9956, 2012.

[43] Y. Estévez-Martínez, C. Velasco-Santos, A.-L. MartínezHernández et al., "Grafting of multiwalled carbon nanotubes with chicken feather keratin," Journal of Nanomaterials, vol. 2013, article 38, 2013.

[44] M. A. Atieh, O. Y. Bakather, B. Al-Tawbini, A. A. Bukhari, F. A. Abuilaiwi, and M. B. Fettouhi, "Effect of carboxylic functional group functionalized on carbon nanotubes surface on the removal of lead from water," Bioinorganic Chemistry and Applications, vol. 2010, Article ID 603978, 9 pages, 2010.

[45] T. Belin and F. Epron, "Characterization methods of carbon nanotubes: a review," Materials Science and Engineering B, vol. 119, no. 2, pp. 105-118, 2005.

[46] S. Vidhate, L. Innocentini-Mei, and N. A. D’Souza, "Mechanical and electrical multifunctional poly (3-hydroxybutyrate-co-3hydroxyvalerate)-multiwall carbon nanotube nanocomposites," Polymer Engineering \& Science, vol. 52, no. 6, pp. 1367-1374, 2012.

[47] M. Lai, J. Li, J. Yang, J. Liu, X. Tong, and H. Cheng, "The morphology and thermal properties of multi-walled carbon nanotube and poly (hydroxybutyrate-co-hydroxyvalerate) composite," Polymer International, vol. 53, no. 10, pp. 1479-1484, 2004.

[48] J. Yang, C. Wang, K. Wang et al., "Direct formation of nanohybrid shish-kebab in the injection molded bar of polyethylene/ multiwalled carbon nanotubes composite," Macromolecules, vol. 42, no. 18, pp. 7016-7023, 2009.

[49] T. J. Mason and J. P. Lorimer, Applied Sonochemistry: The Uses of Power Ultrasound in Chemistry and Processing, Wiley-VCH, Weinheim, Germany, 2002.

[50] A. V. Mohod and P. R. Gogate, "Ultrasonic degradation of polymers: effect of operating parameters and intensification using additives for carboxymethyl cellulose (CMC) and polyvinyl alcohol (PVA)," Ultrasonics Sonochemistry, vol. 18, no. 3, pp. 727-734, 2011. 

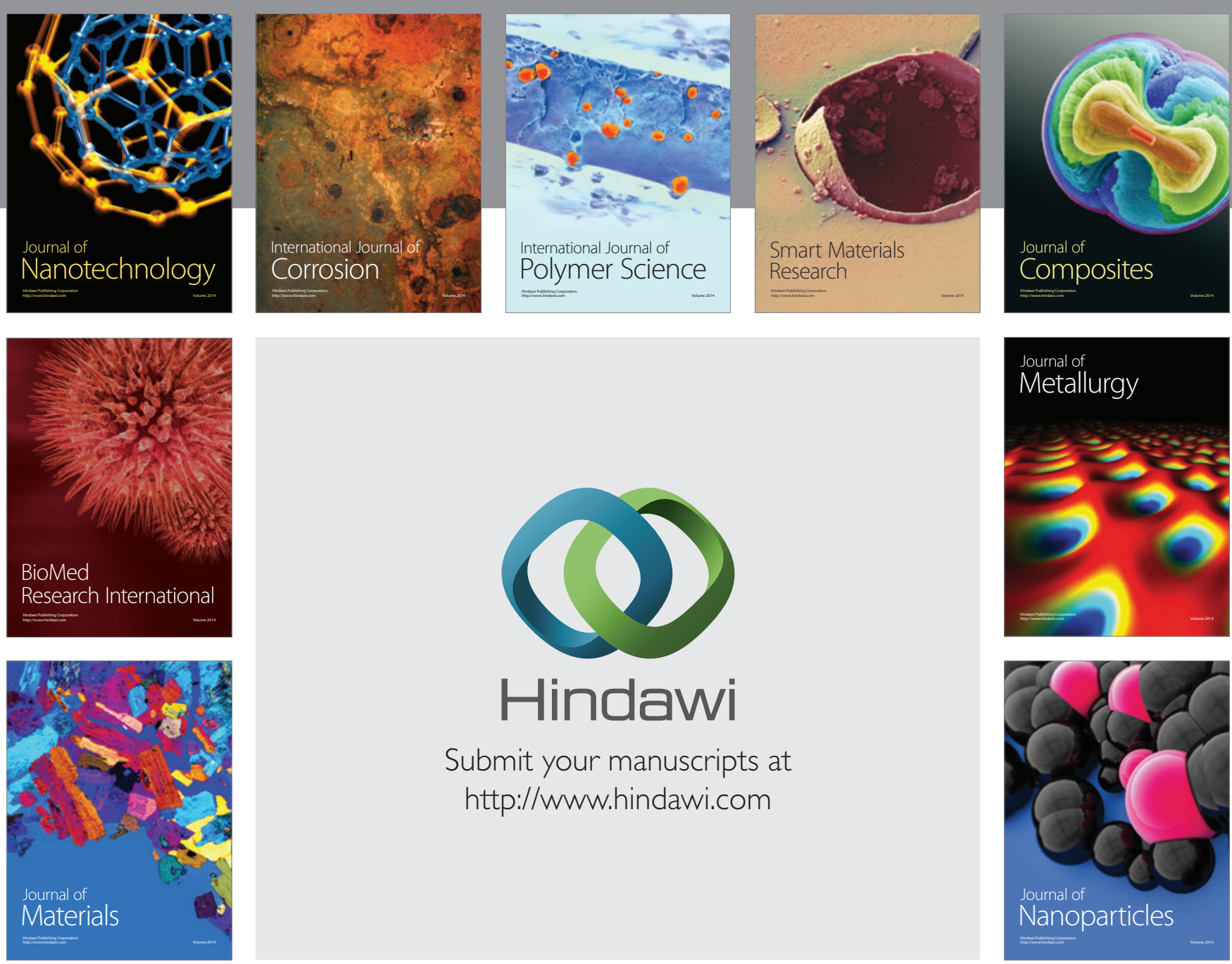

Submit your manuscripts at http://www.hindawi.com
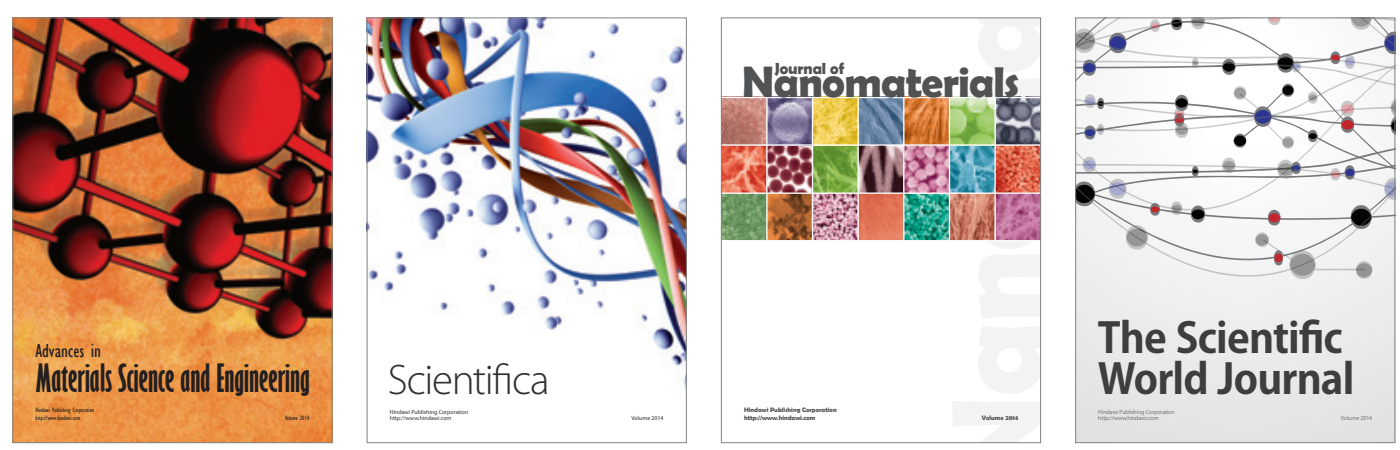

\section{The Scientific World Journal}
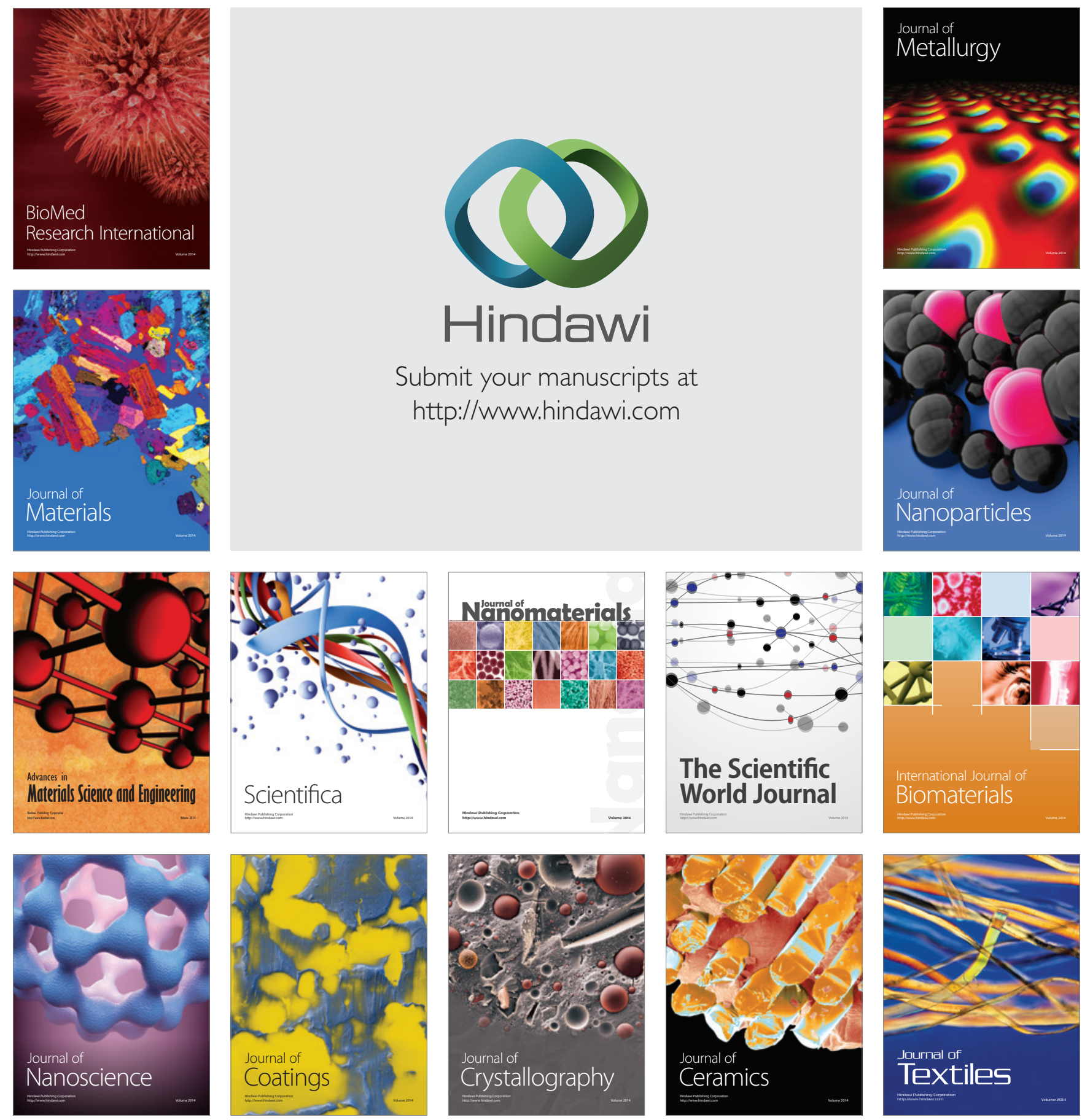